\title{
MFA-Azubis
}

\section{Diese Regeln gelten für die Praxis}

\author{
Gerade erst hat das neue Ausbildungsjahr in den Praxen begonnen. \\ Dabei gibt es nicht nur bei den Lehrinhalten für die Teams einiges zu \\ beachten.
}

$\mathrm{D}$ ie Ausbildung zur Medizinischen Fachangestellten (MFA) ist vor allem bei Frauen nach wie vor beliebt: Im vergangenen Jahr rangierte die Ausbildung in den Praxen bei ihnen auf Platz zwei der 25 am häufigsten besetzten Berufe, wie der Datenreport des Bundesinstituts für Berufsbildung (BIBB) zeigt. Gerade für Praxen, die sich neu im Ausbildungsgeschehen tummeln, gibt es einiges zu beachten.

Die angehenden MFA sind nicht nur in den Betriebsabläufen, der Assistenz des Arztes und der Verwaltung sowie Abrechnung zu schulen. Längst gehören Themen wie Marketing und Kommunikation sowie das Qualitätsmanagement zu den festen Bestandteilen der Ausbildung. Gleichzeitig müssen die Azubis in Informationstechnik sowie Datenschutz und Datensicherheit fit gemacht werden.

\section{Eigener Ausbildungsplan ist Pflicht für jede Praxis}

Die Praxen sind dazu verpflichtet, für ihre Azubis einen Ausbildungsplan zu erstellen, der alle Lehrinhalte der Berufsausbildungsverordnung enthält. Orientierung bieten die Musterausbildungspläne der jeweiligen Ärztekammern. Diese geben in der Regel bereits in tabellarischer Form die Lehrinhalte samt Zeitpunkt und Zeitraum für die Vermittlung vor. Das ist wichtig, weil die angehenden MFA bei einer Zwischenprüfung ihren Lernstand nachweisen müssen.

Nicht vernachlässigen sollten Ärzte das Berichtsheft beziehungsweise den schriftlichen Ausbildungsnachweis, den die Azubis führen müssen. Dieser ist zum einen Voraussetzung, um für die
Abschlussprüfung zugelassen zu werden, zum anderen gibt er dem Praxischef Aufschluss über den Wissensstand der Azubis. Er dient auch als Beweismittel, wenn bei einer nicht bestandenen Prüfung die Azubi behauptet, der von ihr in der Prüfung abverlangte Stoff sei in der Ausbildung nicht vermittelt worden, erklärt die Landesärztekammer Hessen in einem Merkblatt. Die Ausbildungsordnung schreibt vor, dass die Azubis Gelegenheit haben müssen, das Berichtsheft während der Ausbildungszeit - also während der Arbeitszeit - zu führen.

Hilfreich für die Azubis ist es darüber hinaus, wenn sie einen festen Ansprechpartner aus dem Praxisteam zur Seite gestellt bekommen. Damit die Azubis früh eigenständiges und verantwortungsvolles Arbeiten lernen, empfiehlt es sich, ihnen eigene Projekte zu geben, etwa im Rahmen des Qualitätsmanagements oder beim Patienten-Recall. Zusätzlich können Fortbildungseinheiten in Teambesprechungen - in denen mit wechselnder Moderation konkrete Fälle gemeinsam erörtert werden - dazu beitragen, die Angst vor der Prüfung zu nehmen.

\section{Auch für die Berufskleidung trägt die Praxis die Kosten}

Neben den Lehrinhalten sind aber auch die vertraglichen und finanziellen Rahmenbedingungen geregelt: Die Probezeit sollte zwischen einem und vier Monaten betragen. Sie darf dabei nicht länger als vier Monate sein. Nach dem Berufsbildungsgesetz ( $\$ 17$ Abs. 1) ist den angehenden MFA eine angemessene Ausbildungsvergütung zu zahlen. Wer sich am Gehaltstarifvertrag für MFA orientiert, macht nichts falsch. Der Tarifvertrag ist allerdings nur bindend für Arbeitgeber und Azubis, die den beiden Tarifparteien angehören. Das Bundesarbeitsgericht hat jedoch in einem $\mathrm{Ur}$ teil entschieden, dass eine angemessene Vergütung maximal $20 \%$ unter Tarif liegen darf (Az.: 9 AZR 108/14). Derzeit gibt es nach Tarif für die Azubis in den Praxen im ersten Ausbildungsjahr $730 €$ pro Monat.

Greift die Tarifbindung, dann haben die Azubis auch Anspruch auf ein 13. Monatsgehalt sowie die Möglichkeit zur betrieblichen Altersversorgung nach Maßgabe des Tarifvertrags zur betrieblichen Altersversorgung und Entgeltumwandlung. Nimmt eine Auszubildende die Altersvorsorge in Anspruch, fließen dort auch die vermögenswirksamen Leistungen ein, insgesamt stehen der MFA laut Tarifvertrag nach der Probezeit $53 €$ monatlich für den Vertrag zur Altersvorsorge zu.

Die Praxis muss auch die Berufskleidung stellen und die Reinigungskosten übernehmen. In der Regel übernimmt sie zudem die Kosten für die Nutzung öffentlicher Verkehrsmittel für den Weg zwischen Praxis und Berufsschule. Teilweise gibt es aber auch Förderungen durch die einzelnen Bundesländer. Hier lohnt es sich, bei der Ärztekammer nachzufragen. Die Kosten für überbetriebliche Lehrgänge trägt ebenfalls die Praxis. Hier können die Azubis aber mitunter an den Verpflegungskosten beteiligt werden.

Für alle Praxen, die noch auf der Suche nach einer Azubi sind: Zwar liegt der Einstellungstermin offiziell zwischen dem 1. August und 1. September. Es ist aber eine verspätete Einstellung bis zum 31. Dezember möglich. Allerdings mit der Konsequenz, dass sich laut der Landesärztekammer Hessen auch die Abschlussprüfung nach hinten verschiebt.

Rebekka Höhl 